\title{
Receptive-field construction in cortical inhibitory interneurons
}

Harvey A. Swadlow \& Alexander G. Gusev

Nat. Neurosci. 5, 403-404 (2002)

We introduced an error in preparing this article for press. The fifth sentence of the fourth paragraph, "Notably, each of the TC neurons showed strong directional selectivity, but these preferred directions differed over a range of $13^{\circ}$," should have read "over a range of $135^{\circ}$."

\section{Thalamcortical optimization of tactile processing according to behavioral state}

Miguel A.L. Nicolelis \& Erika E. Fanselow

Nat. Neurosci. 5, 517-523 (2002)

The title of this article contained a typographical error. It should have read:

Thalamocortical optimization of tactile processing according to behavioral state

\section{corrigendum}

\section{Progressive induction of caudal neural character by graded Wnt signaling}

Ulrika Nordström, Thomas M. Jessell and Thomas Edlund

Nat. Neurosci. 5, 525-532 (2002)

The authors wish to correct the phrase "rostral-to-caudal shift" on page 528, which should read "rostrocaudal shift". The error occurs twice on this page. 